\title{
Analysis of the effectiveness of wound closure techniques among surgeons and emergency physicians in Mecca Hospitals: Retrospective Cohort Study.
}

Jeehad Mansour Felemban ( $\sim$ Jehad-1-@hotmail.com )

Umm Al-Qura University College of Medicine https://orcid.org/0000-0002-7274-8451

Kenan Binyaseen

Umm Al-Qura University College of Medicine

Waleed Almalki

Umm Al-Qura University College of Medicine

Fahad Altowairqi

Umm Al-Qura University College of Medicine

Amer Sakhakhini

Umm Al-Qura University College of Medicine

Ziyad Alharbi

King Abdullah Medical City

\section{Research note}

Keywords: Cohort Study, Effectiveness, Emergency physicians, Laceration closure, Skin suture, Suture techniques, Suture methods, Surgeons, Wound closure, Wound suture

Posted Date: July 30th, 2020

DOI: https://doi.org/10.21203/rs.3.rs-35558/v2

License: (c) (i) This work is licensed under a Creative Commons Attribution 4.0 International License. Read Full License 


\section{Abstract}

Objective: Wound suturing is a procedure performed at many medical specialties. This study aims to analyze the effectiveness of wound closure techniques by recognizing the common suturing techniques and materials used in different types of wounds among surgeons and emergency physicians in Mecca hospitals and assessing their awareness about the factors contributing to scars formation and improper wound healing.

Result: Choosing the simple interrupted technique exceeded $50 \%$ among all other types of studied wounds. The other most common techniques among participants were the subcuticular and vertical mattress, respectively, also using stapler was limited to some wound types. This study showed a high level of awareness among participants with significant $p$-values $(P<0.05)$ of Strongly agree and Agree regarding the essential factors affecting wound healing and scars formation. The result gives an insight into the most common suture techniques and materials used in wound closure. There are wide variations in selecting these techniques and materials among the participants. Also, it showed that there is good awareness among participants about factors that lead to scar formation.

\section{Introduction:}

Wound suturing, and laceration closure are frequent procedures performed at many surgical and Emergency departments. Different suturing techniques used to treat patients undergoing any surgical closure such as skin suture, adipose, tendon, and muscle, either wound injury conditions or surgical operations. Several suture techniques and materials are available for surgeons and medical practitioners to repair skin and any wound defects. The selection of which technique and material should be used depends on many different variables that affect the outcome of the procedure. Many varieties of suturing materials and needles are available. However, the location of the lesion, type, thickness of the tissue at that location, and amount of tension exerted on the wound determine the choice of sutures and needles (1). Several studies compared different techniques according to specific variables and their effect on the outcomes. Some of these variables are wound complications, speed of repair, cosmoses, patient and physician satisfaction, and cost of reparation (2). The proper suturing techniques depend mainly on eliminating dead space in subcutaneous tissues and minimizing tension that causes wound separation. Also, involving correct wound placement concerning relaxed tension lines (3). Some complications can result from suture procedures such as scars formation, approximately 100 million people develop scars yearly after trauma and elective surgery in middle-income countries, while $15 \%$ of this population will require surgical intervention for their scars due to aesthetic considerations (4). Many studies compared different suturing techniques. A study investigated different suture techniques for patient satisfaction, wound healing, and treatment cost in patients with scalp laceration. It found that there are many alternatives such as adhesions and staples that have more advantages than simple suture $(5,6)$. Other studies showed that cosmetic pleasing and patient satisfaction, which they are significantly better in the 
running horizontal matters and running subcuticular sutures technique than the traditional simple interrupted, are considered essential factors that assess the effect of the suture technique. $(7,8,9)$. Another randomized controlled trial reviewed subcuticular versus interrupted skin suturing. It compared the superficial incisional surgical site infection rate after cesarean section (CS) in obese women. The result was, "Subcuticular skin closure during CS was significantly associated with better short-term cosmetic outcome, less skin closure time." (10)

This study aims to analyze the effectiveness of wound closure techniques among surgeons and emergency physicians in mecca city hospitals, Saudia Arabia, and to assess their awareness about the factors of scar formation and improper wound healing. It fulfilled that by realizing the common suturing methods and the common types and materials used in the closure of Eyelids wound, Scalp wound, Facial wound, Breast wound, Skin wound (other than those mentioned above), and Fascial Plication.

\section{Method:}

A retrospective cohort study was conducted among general surgery (GS) and Emergency (EM) physicians. They were enrolling and preforming any suture procedure aiming to investigate their practice of suturing on their patients. They focused on patients who underwent any wound closure procedure for different skin wounds in the last year in the seven various hospitals of Mecca, Saudi Arabia. Research committee approval was obtained, and a sample of 172 surgeons and EM physicians were calculated to give us $80 \%$ power with an alpha level of $5 \%$. A random generator software was used for randomization. A stratified random sampling technique was applied to an equal number of surgeons and EM physicians from both departments of the seven different hospitals of Mecca City. They were asked about their practice regarding their suturing technique and materials for the different wound types. This study has assessed its participants' awareness of how much these factors will substantially impact the rate of scar formation. During two months of period, the data was collected using a self-administered questionnaire on an electronic sheet that included demographic information, suture techniques, and materials about different wound types, and the factors affect the rate of scars. Patients with certain conditions that could affect the measuring factors were excluded. So, patients with hematological disorders, autoimmune disease, liver diseases, diabetes mellitus, thyroid diseases, renal diseases, and immunodeficiency were not included. Verbal and written consent was obtained from all participants. However, those who did not participate in filling up the survey were respected. Statistical analysis was performed using SPSS software. Sample descriptive statistics (mean, median, SD range) were applied to demographic data and different suturing techniques and materials. Chi-square and T-test were used to assess the level of awareness for surgeons and ER physicians about the factors that have a role in the rate of scar formation, which was evaluated via a Likert scale.

\section{Results:}

Our study surveyed 172 participants, and we summarized the finding in Figure1, which shows the common types of suturing techniques. Most of the responses were about using the simple interrupted 
technique in different kinds of wounds mentioned in the study. Moreover, the number of participants who choose the simple interrupted technique among other types of techniques exceed $50 \%$ for all types of the mentioned wounds especially for the scalp and eyelids wound where $77.9 \%$ of participants select the simple interrupted for these types of wounds, also $80.8 \%$ of them select it for buttocks wounds. The second common technique is the subcuticular technique, whereas $42.4 \%$ of practitioners select it for breast wound and $33.1 \%$ for the facial wounds. Moreover, the third was a vertical mattress where $14.5 \%$ of participants used it for buttocks wound. However, the stabler selection was limited to some wound types, where $5.2 \%$ of participants select it for scalp wounds and $8.7 \%$ for skin wound (other than mentioned).

Figure2: It shows the uses of different suturing material types, $23 \%$ of participants use Monocryl for eyelids wounds, and $6 \%$ for breast wounds. Polypropylene is used in different types of wounds $9 \%$ of participants using it for scalp wounds and $8 \%$ for eyelids wounds. Silk suture was one of the most commonly used suture materials among our participants, especially for facial wounds $13 \%$. Fascial Plication was often closed by Polylactic acid sutures, which represent $10 \%$ of its uses. There is also the polyglycolic acid, which was commonly used over the other materials.

Figure3: the results showed a high level of awareness among participants regarding all the mentioned factors of scars formation. Whereas the P-value were less than 0.01 for wound position, wound tension, using the proper suturing technique, using the proper suturing material, and nutritional deficiencies. And the participants who chose strongly agree and agree were more than those who choose the other options. Also, a high level of awareness has been shown with $P$ value equal to 0.017 for the factor of systemic diseases and 0.044 for age of the patient.

\section{Discussion:}

The present study was conducted to analyze wound closure techniques' effectiveness by recognizing the common suturing technique and the conventional type and materials of sutures used in different wound types by surgeons and emergency physicians in Mecca Hospitals. The finding represents no particular methods used from all participants in their suturing practice regarding the specific types of wounds mentioned in the study. Every surgeon and EM physician selected and preferred some methods in wound closure over other methods, which may affect wound closure outcomes among practitioners. However, the previous studies also showed that many suture techniques used nowadays, some of them used more frequently than others due to their advantages and suitability. The most common and fundamental technique of wound closure is a simple interrupted suture (3). This is similar to in our participants, whereas most of them chose the simple interrupted technique among other types of techniques with a percentage exceeded $50 \%$ of all types of the mentioned wounds. Consequently, this led us to recognize the urgent need for increasing education and awareness for selecting this type of 
technique just in the skin's designated site and avoiding other sites, especially in the face or breast. The interrupted sutures are easy to place and possess greater tensile strength. (3) However, the subcuticular suture technique, which resembles the second most common technique in our study, is used more frequently in the breast and facial wound. literature showed that this technique is used more than the traditional simple interrupted to enhance the cosmetic result and proved its effectiveness, it is also useful for closing wounds with equal tissue thickness in which virtually no tension exists. $(7,10)$ Another suture technique is vertical mattress suture, which was the third most common technique in this study and used more frequently in the buttock wound. The literature also showed that it is a widespread technique and useful in maximizing the wound eversion, reducing dead space, and minimizing tension across the wound. (3) Staplers' use in the closure of scalp wounds has more advantages than simple interrupted suture in regard to patient satisfaction, wound healing, and concerning cost. (5) however, in our study, $5.20 \%$ of the participants choose staplers in scalp wounds, which are a deficient percentage compared to $77.90 \%$ who are choosing the simple interrupted technique which is not considered as the best technique for scalp wound according to the previous studies. (6) Moreover, many different suturing materials can be used at many various types of wounds, apart from the most suitable types of material. The selection of excellent material in wound closure may contribute to satisfactory outcomes in wound healing processes, cosmetic pleasing, or any other expected outcomes. This study has not shown certain types of material that are used by all participants in their practice. However, some materials are more frequently used than others, such as polyglycolic acid, Silk, Polylactic acid, Monocryl, Polypropylene, and catgut. Therefore, there must be an awareness and intensive education regarding the proper use of material with the area and the wound's level because it seems there is a wide verity of using material without strict guidelines. On the other hand, this study has evaluated the awareness about the factors of scars formation and the improper healing among surgeons and ER physicians during their practice of wound closure. The results showed a high level of awareness with significant $p$ values for all the study's mentioned factors. These findings may reflect a better practice attitude regarding scar formation. Still, the lack of evidence and guidelines for selecting the proper type of suture technique and materials leads to some limitations in analyzing the most common suture technique and materials' effectiveness. Also, there is no data about actual outcomes following skin closure at Mecca's seven different hospitals. This study depends on its comparison upon various previous studies that reviewed a few methods mentioned, whereas the other methods have not been reviewed yet. Our recommendation is to consider new criteria for selecting the most proper type of suture technique and material that can be applied to all the different types of wounds by surgeons and EM physicians to have the optimum outcomes in any wound closure procedure. Moreover, considering a new monitoring system for the outcomes following skin closure procedure to acquire the optimum outcomes and improve the suturing practice quality.

\section{Conclusions:}

This study provides an insight into the most common suture methods used among surgeons and emergency physicians in Mecca Hospitals. The results suggest different suture methods and materials used in various types of wound closure; some of the techniques and materials are better and frequently 
used than others in different wound conditions. Besides, there are wide variations in selecting these techniques and materials among surgeons and emergency physicians, reflecting on the outcome result of wounds suture among them. However, intensive education and awareness must be offered regarding the proper use of techniques and materials in skin suturing and closure. At the same time, it has been noticed that there is good awareness among participants about factors that lead to scar formation and improper wound healing significantly.

\section{Limitation:}

This study's limitations were represented in the potential for information bias during data collection in receiving answers by using an online questionnaire rather than submitting the questionnaire physically, and this study was conducted among participants from limited hospitals.

\section{Declaration:}

\section{Research approval:}

was obtained from Institutional Review Board- King Abdullah Medical City - Mecca - Saudi Arabia, IRB number $19-588$.

Consent for publication: written consent was obtained from all participants.

Availability of data: All data generated or analyzed during the current study are included in this published article and its supplementary information files.

Competing interest: Not applicable

Funding: Not applicable

\section{Authors conurbations:}

Ziyad Alharbi and Jeehad Felemban contributed to the conception and the design of the study

Kenan Binyaseen and Waleed S Almalki wrote the manuscript draft.

Amer Sakhakhini, Fahad Altowairqi, and Jeehad Felemban contributed to data collection, analysis, and data interpretation.

Ziyad Alharbi and Jeehad Felemban wrote the final form of the manuscript.

All authors corrected and approved the manuscript.

Acknowledgments: The authors would like to extend their appreciation to Umm Alqura University Mecca - Saudi Arabia, Institutional Review Board- King Abdullah Medical City - Mecca - Saudi Arabia. I 
would like to thank my other coworkers due to their contributions to this study.

\section{Abbreviations:}

GS: General surgery

EM: Emergency Medicine

CS: Cesarean section

\section{References:}

1. J Mackay-Wiggan. Suturing Techniques Technique: General Principles, Placement of Specific Suture Types, Alternative Methods of Wound Closure [Internet]. Emedicine.medscape.com. [cited 2019]. Available from: https://emedicine.medscape.com/article/1824895-technique.

2. K Mackay-Jones Emerg Med J. Towards evidence-based emergency medicine Best BETs from the Manchester Royal Infirmary. 1 July 2002. [online] Available at: https://emj.bmj.com/content/33/10/741.1

3. Kuduro MH, Pai SB, Sripathi H, Prabhu S. Sutures, and suturing techniques in skin closure. Indian J Dermatol Venereol Leprol .2009;75:425-34. Available from: http://www.ijdvl.com/text.asp? 2009/75/4/425/53155

4. Al-Shaqsi, S. and Al-Bulushi, T., 2016. Cutaneous Scar Prevention And Management: Overview Of Current Therapies.

5. Cemil, K., Yusuf, C.,evik. Comparison of Different Suture Techniques. Journal of Clinical and Analytical Medicine. (2015). https://doaj.org/article/6930d678c39b49af89535611750dcdcf

6. Kanegaye, J., Vance, C., Chan, L., \& Schonfeld, N. Comparison of skin stapling devices and standard sutures for pediatric scalp lacerations: A randomized study of cost and time benefits. The Journal of Pediatrics. (1997); 130(5), 808-813. DOI: 10.1016/s0022-3476(97)80025-x

7. Liu X, e. Aesthetic outcome and complications of simple interrupted versus running subcuticular sutures in facial surgery: A randomized controlled trial. 2017 Nov;77(5):911-919. - PubMed NCBI. [online] Ncbi.nlm.nih.gov. Available at: https://www.ncbi.nlm.nih.gov/pubmed/28728869

8. Moody BR, e. The enhanced cosmetic outcome with running horizontal mattress sutures. 2005 Oct;31(10):1313-6 Available at https://www.ncbi.nlm.nih.gov/pubmed/16188185.

9. Javadi S M R, Kasraianfard A, Ghaderzadeh P, Khorshidi H R, Moein A, et al. Comparison of Subcuticular and Interrupted Suturing Methods for Skin Closure After Appendectomy: A Randomized Controlled Trial, Iran Red Crescent Med J. 2018 ; 20(1):e14469. 
10. Ibrahim, M., Moustafa, G., Al-Hamid, A., \& Hussein, M. Superficial incisional surgical site infection rate after cesarean section in obese women: a randomized controlled trial of subcuticular versus interrupted skin suturing. Archives Of Gynecology And Obstetrics. (2013); 289(5), 981-986. DOI: 10.1007/s00404-013-3098-z

\section{Figures}

Figure 1

\section{Types of Suture Technique used in diffrent types of wounds}

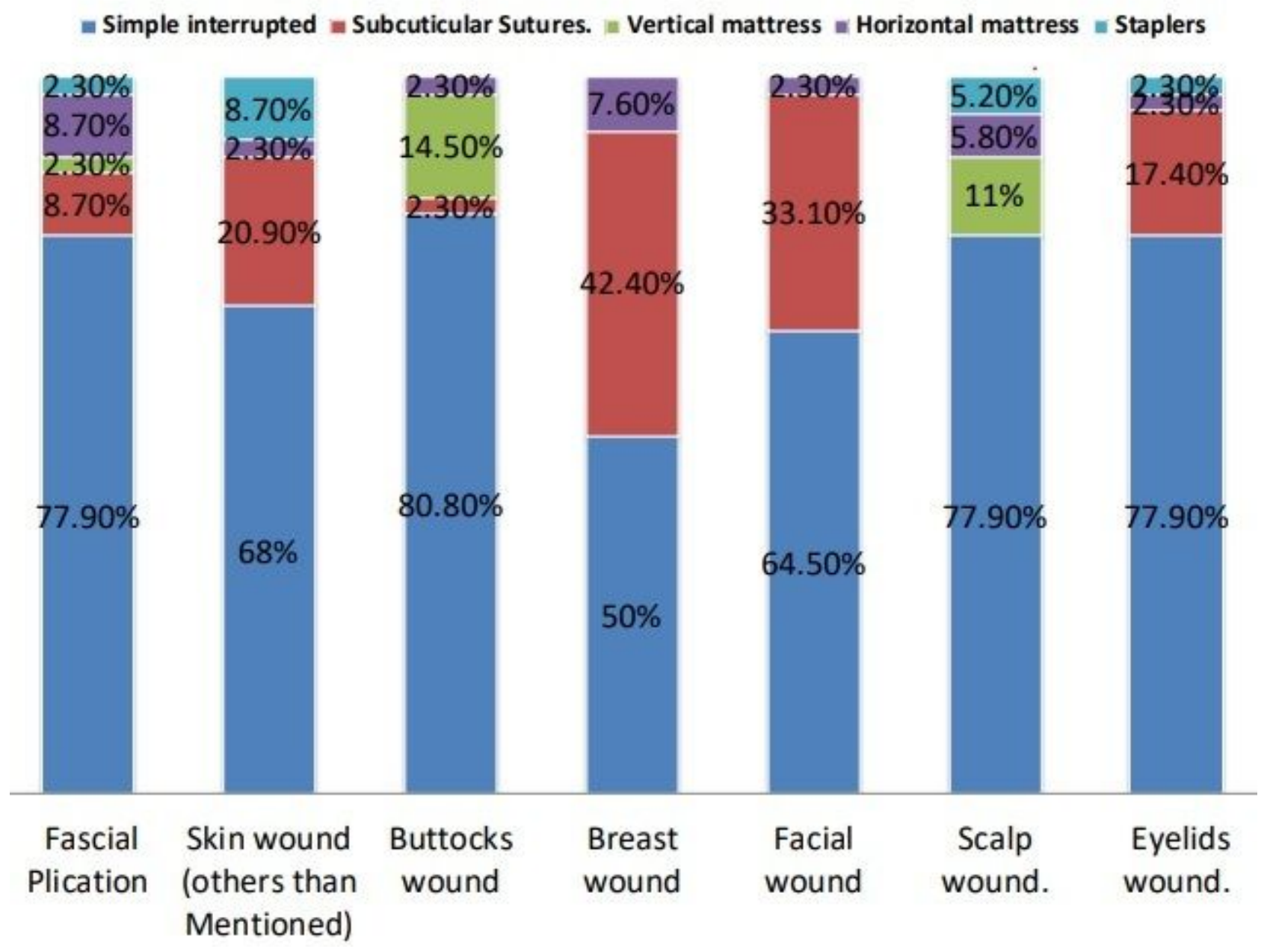

Figure 1

The different types of suturing techniques regarding certain types of wounds. 
Figure 2

\section{types of suturing material used in different types of wounds}

$\begin{array}{lll}\text { Eyelids wounds } & \text { Buttocks wounds } & \text { Scalp wounds } \\ \text { Fascial Plication } & \text { facial wounds } & \text { breast wounds }\end{array}$

- skin wounds (other than mentioned)

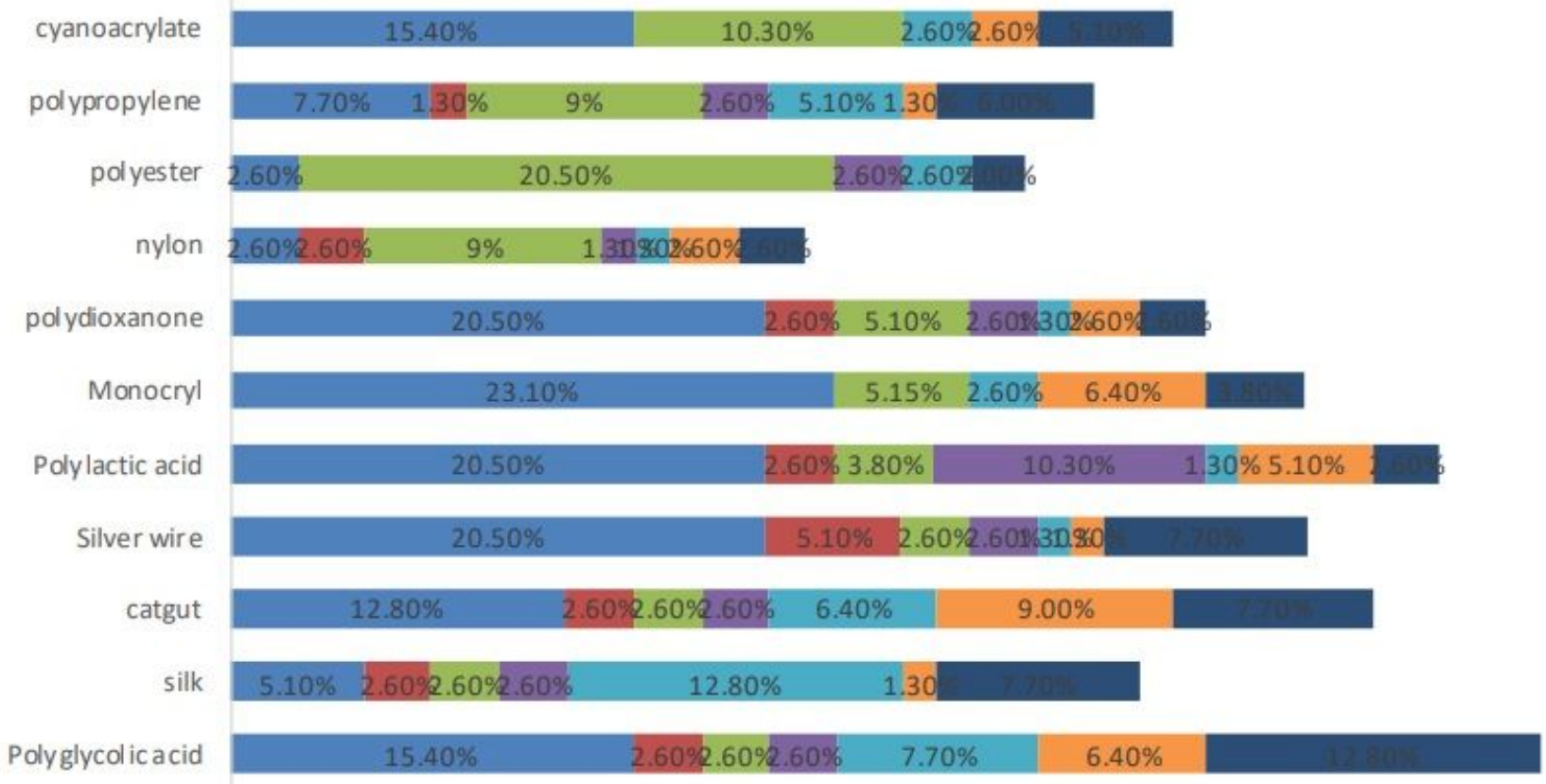

\section{Figure 2}

The different types of suturing material regarding certain types of wounds.

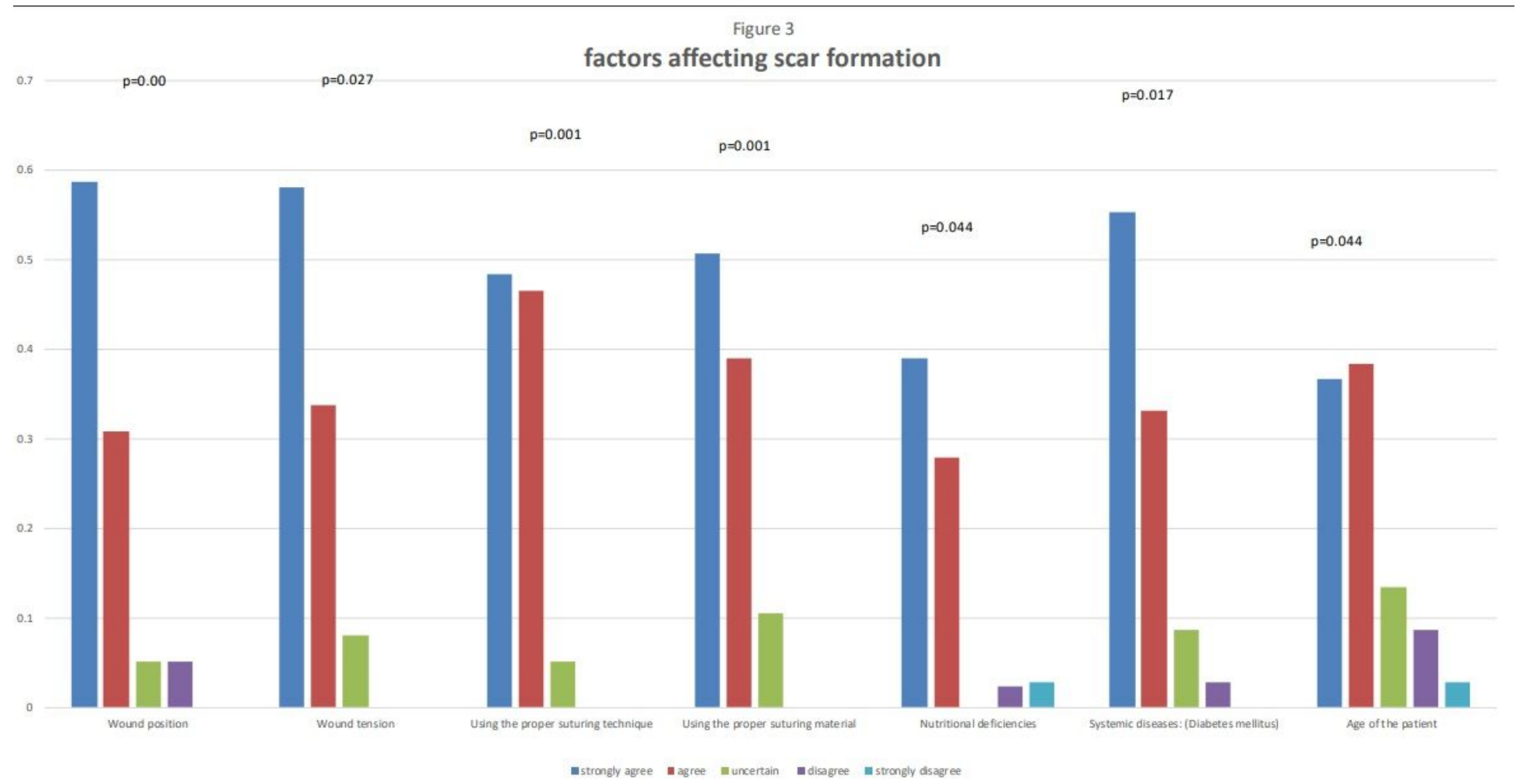


Figure 3

The factors that affect scars formation.

\section{Supplementary Files}

This is a list of supplementary files associated with this preprint. Click to download.

- AllData.xlsx

- AdditionalFiles.docx 